\title{
A classification of ordinals up to Borel isomorphism
}

by

\section{Su Gao, Steve Jackson and Vincent Kieftenbeld (Denton, TX)}

\begin{abstract}
We consider the Borel structures on ordinals generated by their order topologies and provide a complete classification of all ordinals up to Borel isomorphism in ZFC. We also consider the same classification problem in the context of AD and give a partial answer for ordinals $\leq \omega_{2}$.
\end{abstract}

1. Introduction. Recall that the order topology on a linearly ordered set $X$ is generated by the subbase of open rays $(x, \rightarrow)=\{y \in X: x<y\}$ and $(\leftarrow, y)=\{x \in X: x<y\}$ for $x, y \in X$. It is the most natural topology on ordinals. When we speak of an ordinal as a topological space we always assume that it has the order topology.

A complete classification of ordinals up to homeomorphism is known ([1]; an independent proof was given in [5]). Specifically, given an arbitrary ordinal a complete homeomorphism invariant for its order topology can be computed from its Cantor normal form. Benedikt Löwe proposed to study the similar classification problem for ordinals up to Borel isomorphism. He asked whether the Cantor normal form still provides a complete invariant. Since for example all countable ordinals are Borel isomorphic, Borel isomorphism is a genuinely more general notion of equivalence than homeomorphism.

In this paper we give a complete classification of all ordinals up to Borel isomorphism. It turns out that the computation of the complete invariants is not related to the Cantor normal form of the ordinals and is in fact somewhat simpler. To state our main theorem precisely, we define a cardinal $\kappa(\alpha)$ for any given ordinal $\alpha$ as follows. For an ordinal $\alpha$, let $\kappa(\alpha)=0$ if $|\alpha|$ is singular or countable, and otherwise let $\kappa(\alpha)$ be the largest cardinal such that $|\alpha| \cdot \kappa(\alpha) \leq \alpha$.

2000 Mathematics Subject Classification: Primary 03E10, 54H05; Secondary 03E60, 06F30.

Key words and phrases: order topology, ordinals, Borel isomorphism, Borel $\sigma$-algebra.

The first author acknowledges the support of U.S. NSF grant DMS-0501039. 
THEOREM 1.1. Let $\alpha$ and $\beta$ be ordinals. Then $\alpha$ is Borel isomorphic to $\beta$ iff $|\alpha|=|\beta|$ and $\kappa(\alpha)=\kappa(\beta)$.

Note that the above main theorem will be proved in ZFC, in particular with essential use of $A C$ in the proof. This is in contrast with the classification of ordinals up to homeomorphism, which can be done in ZF only (this is easier to see from the presentation of [5]). Not much of our discussions on the Borel structures of ordinals can survive in ZF. It thus seems to be very interesting to consider the same classification problem in the context of $A D$ and to see how different the complete invariants would be. On this we have the following partial result.

THEOREM $1.2(\mathrm{ZF}+\mathrm{AD})$. All uncountable ordinals $<\omega_{2}$ are Borel isomorphic.

This paper is organized as follows. In Section 2 we review some preliminaries on the Borel structures generated by the order topologies on ordinals. In particular we give a characterization of Borelness for subsets of ordinals which will be useful in further research. In Section 3 we give the proof of Theorem 1.1. In Section 4 we work under determinacy and prove Theorem 1.2 .

We would like to thank Benedikt Löwe and Dan Mauldin for useful discussions on the subject.

2. Preliminaries. Recall that the Borel structure of any topological space is the $\sigma$-algebra generated by its open sets, i.e., the smallest $\sigma$-algebra that contains all the open sets and is closed under complements and countable unions. Also all Borel sets appear in the Borel hierarchy, which is defined by induction on $\alpha<\omega_{1}$ as follows:

$\Sigma_{1}^{0}=$ all open sets,

$\boldsymbol{\Pi}_{\alpha}^{0}=$ all complements of $\boldsymbol{\Sigma}_{\alpha}^{0}$ sets,

$\boldsymbol{\Sigma}_{\alpha}^{0}=$ all countable unions $\bigcup_{n \in \mathbb{N}} A_{n}$, where $A_{n} \in \boldsymbol{\Pi}_{\alpha_{n}}^{0}$ for some $\alpha_{n}<\alpha$, $\Delta_{\alpha}^{0}=\Sigma_{\alpha}^{0} \cap \Pi_{\alpha}^{0}$

The following proposition records the basic facts about these levels of the Borel hierarchy which are true in any topological space.

Proposition 2.1. In any topological space the following hold. $\boldsymbol{\Sigma}_{\alpha}^{0} \subseteq \boldsymbol{\Pi}_{\beta}^{0}$ for $\alpha<\beta$, and similarly, $\boldsymbol{\Pi}_{\alpha}^{0} \subseteq \boldsymbol{\Sigma}_{\beta}^{0} . \boldsymbol{\Sigma}_{\alpha}^{0}$ is closed under countable unions and $\boldsymbol{\Pi}_{\alpha}^{0}$ under countable intersections. $\boldsymbol{\Sigma}_{\alpha}^{0}$ is closed under finite intersections and $\boldsymbol{\Pi}_{\alpha}^{0}$ under finite unions for all $\alpha \neq 3$. If $2 \leq \alpha \leq \beta$, then $\boldsymbol{\Sigma}_{\alpha}^{0} \subseteq \boldsymbol{\Sigma}_{\beta}^{0}$ and $\boldsymbol{\Pi}_{\alpha}^{0} \subseteq \boldsymbol{\Pi}_{\beta}^{0}$. 
Proof. All of the statements are immediate from the definitions except perhaps the closure of $\Sigma_{\alpha}^{0}$ under finite intersections for $\alpha \neq 3$. To see this, suppose $A, B \in \Sigma_{\alpha}^{0}$, say $A=\bigcup_{n} A_{n}, B=\bigcup_{m} B_{m}$, where $A_{n} \in \Pi_{\alpha_{n}}^{0}$, $B_{m} \in \boldsymbol{\Pi}_{\beta_{m}}^{0}$ and $\alpha_{n}, \beta_{m}<\alpha$. Then $A \cap B=\bigcup_{n, m}\left(A_{n} \cap B_{m}\right)$. If $\alpha \geq 4$, then $A_{\alpha_{n}}, B_{\beta_{m}}$ both lie in $\boldsymbol{\Pi}_{\delta}^{0}$ where $\delta=\max \left\{\alpha_{n}, \beta_{m}, 3\right\}$. This is because $\boldsymbol{\Pi}_{\alpha}^{0} \subseteq \boldsymbol{\Pi}_{\beta}^{0}$ for $2 \leq \alpha \leq \beta$ and $\boldsymbol{\Pi}_{1}^{0} \subseteq \boldsymbol{\Sigma}_{2}^{0} \subseteq \boldsymbol{\Pi}_{3}^{0}$. Since $\boldsymbol{\Pi}_{\delta}^{0}$ is closed under intersections, $A_{\alpha_{n}} \cap B_{\beta_{m}} \in \boldsymbol{\Pi}_{\delta}^{0}$, and so $A \cap B \in \boldsymbol{\Sigma}_{\alpha}^{0}$. If $\alpha=1$, the result is immediate from the definition of a topology, and if $\alpha=2$ the result follows from the fact that both $A_{\alpha_{n}}, B_{\beta_{m}}$ will be $\boldsymbol{\Pi}_{1}^{0}$, and thus so will be $A_{\alpha_{n}} \cap B_{\beta_{m}}$.

If the underlying space is Polish (completely metrizable and separable) or even just metrizable, then the Borel hierarchy has the usual additional properties such as $\boldsymbol{\Sigma}_{\alpha}^{0} \subseteq \boldsymbol{\Sigma}_{\beta}^{0}$ for $\alpha<\beta$ (and similarly on the $\boldsymbol{\Pi}$-side) and $\boldsymbol{\Sigma}_{\alpha}^{0}, \boldsymbol{\Pi}_{\alpha}^{0}$ are closed under finite unions and finite intersections. In particular, every $\boldsymbol{\Sigma}_{\alpha+1}^{0}$ set is a countable union $\bigcup_{n} A_{n}$ where each $A_{n}$ is $\boldsymbol{\Pi}_{\alpha}^{0}$.

However, these additional facts are no longer true for ordinal spaces. If the underlying space is an uncountable ordinal, then there are always open sets which are not $F_{\sigma}$. Thus in general $\boldsymbol{\Sigma}_{1}^{0} \nsubseteq \boldsymbol{\Sigma}_{2}^{0}$ and $\boldsymbol{\Pi}_{1}^{0} \nsubseteq \boldsymbol{\Pi}_{2}^{0}$. The following observation shows that $\boldsymbol{\Sigma}_{3}^{0}$ is not closed under finite intersections if the underlying space is an ordinal $\geq \omega_{2}$.

Proposition 2.2. There exists an open $U \subseteq \omega_{2}$ and a closed $F \subseteq \omega_{2}$ such that $U \cap F$ is not $\boldsymbol{\Sigma}_{3}^{0}$.

Proof. Let $U=\omega_{2}-\left\{\omega_{1} \cdot \alpha: \alpha<\omega_{2}\right\}$ and let $F$ be the set of all limit ordinals below $\omega_{2}$. Clearly, $U$ is open and $F$ is closed. Suppose $U \cap F$ is $\Sigma_{3}^{0}$, say

$$
U \cap F=\bigcup_{n \in \omega} A_{n} \cup \bigcup_{n \in \omega} B_{n}
$$

where each $A_{n}$ is $\boldsymbol{\Pi}_{1}^{0}$ and each $B_{n}$ is $\boldsymbol{\Pi}_{2}^{0}$. Since $U$ misses a club in $\omega_{2}, U \cap F$ is nonstationary, which in turn implies that each $A_{n}$ is bounded in $\omega_{2}$. Now the union $\bigcup_{n \in \mathbb{N}} A_{n}$ is also bounded in $\omega_{2}$; let $\beta$ be an upper bound. Let $\alpha_{0}$ be the least ordinal such that $\omega_{1} \cdot \alpha_{0} \geq \beta$.

Now consider the copy of $\omega_{1}$ consisting of ordinals in the interval $I=$ $\left(\omega_{1} \cdot \alpha_{0}, \omega_{1} \cdot \alpha_{0}+\omega_{1}\right)$. Our assumption implies that the limit ordinals in $I$ can be written as $\bigcup_{n}\left(B_{n} \cap I\right)$. It follows that the limit ordinals in $\omega_{1}$ can be written as $\bigcup_{n} C_{n}$ where each $C_{n}$ is $\boldsymbol{\Pi}_{2}^{0}$. Since the limit ordinals in $\omega_{1}$ form a club, one of the $C_{n}$ must be stationary. We claim that a stationary $\boldsymbol{\Pi}_{2}^{0}$ subset of $\omega_{1}$ must contain a tail, and this is a contradiction.

Suppose $G=\bigcap_{n \in \omega} G_{n}$ is a stationary $\boldsymbol{\Pi}_{2}^{0}$ in $\omega_{1}$, with all $G_{n}$ open. Each $G_{n}$ is also stationary, and therefore it contains a tail. Since $\operatorname{cof}\left(\omega_{1}\right)>\omega$, a countable intersection of tails is still a tail. Hence, $G$ contains a tail. 
The Borel structures on ordinals have been studied before, e.g., in [8] and [6]. We summarize the known results as well as present the techniques used in the study of this topic. For the convenience of the reader we include some proofs of previously known results here.

Lemma 2.3 (Rao-Rao [8]). Every Borel subset of a limit ordinal either contains or misses a club.

Proof. Every subset of a limit ordinal of cofinality $\omega$ either contains or misses a club. In the case of uncountable cofinality, a countable intersection of clubs is still a club. Hence, the collection of all sets which contain or miss a club is a $\sigma$-algebra containing all closed sets and therefore contains all the Borel sets.

In particular, a stationary and costationary subset of a limit ordinal is not Borel. For $\omega_{1}$, a subset is Borel if and only if it either contains or misses a club [8]. Another characterization of Borel subsets of $\omega_{1}$ was also given in [8], and it was completely generalized by Mauldin in [6], as follows.

TheOREM 2.4 (Mauldin [6]). Every Borel subset of an ordinal can be expressed as a union of countably many sets, each of which is the intersection of an open set and a closed set.

Mauldin's theorem shows that the Borel hierarchy on any ordinal collapses to a rather low level, and every Borel subset of an ordinal is in fact $\boldsymbol{\Delta}_{4}^{0}$. In view of Proposition 2.2 this estimate is optimal.

Below we give another characterization of Borelness of subsets of ordinals. We state the result in a way that encompasses the results in [8] and [6], and provide a self-contained proof. It should be noted, however, that the main ideas and techniques used in the proof are the same as those presented in [8] and [6].

We will use the following simple lemma repeatedly throughout the paper.

Lemma 2.5. Let $X$ be an arbitrary topological space. Suppose $X=$ $\bigcup_{i \in I} U_{i}$, where $\left\{U_{i}\right\}_{i \in I}$ is a family of pairwise disjoint open subsets. Let $\xi<\omega_{1}$ and $B \subseteq X$. Then $B$ is $\boldsymbol{\Sigma}_{\xi}^{0}\left(\right.$ or $\left.\boldsymbol{\Pi}_{\xi}^{0}\right)$ iff for every $i \in I, B \cap U_{i}$ is $\boldsymbol{\Sigma}_{\xi}^{0}$ (respectively $\boldsymbol{\Pi}_{\xi}^{0}$ ) in $U_{i}$.

Proof. A simple induction on $\xi$.

TheOREM 2.6. Let $\alpha$ be an ordinal. Then the following are equivalent:

(1) $B \subseteq \alpha$ is Borel.

(2) $B=\bigcup_{n \in \mathbb{N}}\left(U_{n} \cap F_{n}\right)$, where each $U_{n}$ is open and each $F_{n}$ is closed.

(3) For every limit ordinal $\beta \leq \alpha, B$ contains or misses a club in $\beta$.

(4) For every limit ordinal $\beta \leq \alpha$ and every club $C$ in $\beta, B$ contains or misses a club of $C$. 
Proof. The implication $(1) \Rightarrow(4)$ is immediate from Lemma 2.3. The implications $(2) \Rightarrow(1)$ and $(4) \Rightarrow(3)$ are trivial. It suffices to show $(3) \Rightarrow(2)$. We use induction on $\alpha$. For the base case and the successor case there is nothing to do. We assume that $\alpha$ is a limit. By (3), $B$ contains or misses a club in $\alpha$. For definiteness assume that $B$ misses a club $C$ in $\alpha$. In this case let $\alpha_{i}$, $i<\eta=\operatorname{cof}(\alpha)$, enumerate the elements of $C$ in increasing order. Without loss of generality assume $\alpha_{0}=0$. Then let $U_{i}=\left(\alpha_{i}, \alpha_{i+1}\right)$ for $i<\eta$. Thus we get $\alpha-C=\bigcup_{i<\eta} U_{i}$. Note that (3) is still true for each interval $U_{i}$. Since each $U_{i}$ is a copy of an ordinal $<\alpha$, the inductive hypothesis implies that $B \cap U_{i}$ is a union of countably many sets, each of which is the intersection of an open set with a closed set. Now the proof of Lemma 2.5 implies that

$$
B=B \cap(\alpha-C)=\bigcup_{n \in \mathbb{N}}\left(U_{n} \cap F_{n}\right)
$$

for relatively open $U_{n}$ in $\alpha-C$ and relatively closed $F_{n}$ in $\alpha-C$. Let $C_{n}$ be the closure of $F_{n}$ in $\alpha, U_{-1}=\alpha-C$ and $V_{n}=U_{n} \cap U_{-1}$. Then each $V_{n}$ is open in $\alpha, C_{n}$ is closed in $\alpha, F_{n}=C_{n} \cap U_{-1}$ and

$$
B=\bigcup_{n \in \mathbb{N}}\left(U_{n} \cap F_{n}\right)=\bigcup_{n \in \mathbb{N}}\left(U_{n} \cap C_{n} \cap U_{-1}\right)=\bigcup_{n \in \mathbb{N}}\left(V_{n} \cap C_{n}\right) .
$$

This finishes the proof of the case that $B$ misses a club $C$ in $\alpha$. Suppose alternatively that $B$ contains a club $C$ in $\alpha$; then $B-C$ continues to satisfy (3) and the same argument shows that $B-C$ is a union as in (2). It follows that $B$ is of the same form since $B=(B-C) \cup C$.

Another application of the same technique is the following.

Proposition 2.7. Every Borel subset of $\omega_{1}$ is $\boldsymbol{\Delta}_{3}^{0}$.

Proof. It suffices to show that every Borel subset of $\omega_{1}$ is $\boldsymbol{\Sigma}_{3}^{0}$, and in view of Theorem 2.4 it is enough to show that the intersection of an open set $U$ and a closed set $F$ is $\Sigma_{3}^{0}$. If $U \cap F$ is bounded then it is countable and easily seen to be $\Sigma_{2}^{0}$. Assume $U \cap F$ is unbounded. In particular both $U$ and $F$ are unbounded. If $\omega_{1}-U$ is bounded, then the bounded part of $U \cap F$ is relatively $\boldsymbol{\Sigma}_{2}^{0}$, the unbounded part is relatively closed, thus relatively $\boldsymbol{\Sigma}_{2}^{0}$, hence by Lemma $2.5, U \cap F$ is $\boldsymbol{\Sigma}_{2}^{0}$ in $\omega_{1}$.

If $\omega_{1}-U$ is unbounded, write $U=\bigcup I_{\gamma}$, where the $I_{\gamma}$ are maximal disjoint open intervals. Each $I_{\gamma}$ is homeomorphic to a countable ordinal, hence $U \cap F$ is $\Pi_{2}^{0}$ in $I_{\gamma}$, thus in $U$. Hence, $U \cap F$ is the intersection of an open and a $\boldsymbol{\Pi}_{2}^{0}$ set in $\omega_{1}$, hence $\boldsymbol{\Pi}_{2}^{0}$.

In view of the collapse of the Borel hierarchy our basic Lemma 2.5 can be restated as the following convenient fact for subsets of ordinals. For obvious reasons we will refer to it as the gluing lemma. 
Corollary 2.8 (The gluing lemma). Let $\alpha$ be an ordinal, let $\left\{U_{i}\right\}_{i \in I}$ be a family of pairwise disjoint open sets in $\alpha$, and let $C$ be the closed set $\alpha-\bigcup_{i \in I} U_{i}$. Then a subset $B$ of $\alpha-C$ is Borel in $\alpha$ iff $B \cap U_{i}$ is Borel in $U_{i}$ for every $i \in I$.

We now turn to a review of Borel isomorphisms. Let $X$ and $Y$ be arbitrary topological spaces. A map $f: X \rightarrow Y$ is called Borel measurable (or simply Borel) if for any open set $U$ in $Y, f^{-1}(U)$ is a Borel subset of $X$. Note that the preimage of a Borel set under a Borel map is Borel. $f$ is called a Borel isomorphism if it is a bijection such that both $f$ and $f^{-1}$ are Borel. If there is a Borel isomorphism from $X$ onto $Y$ then we say that $X$ and $Y$ are Borel isomorphic, and denote it by $X \cong_{\mathrm{B}} Y$.

Recall again that if both $X$ and $Y$ are Polish spaces then $X \cong_{\mathrm{B}} Y$ iff there is a Borel injection from $X$ into $Y$ and also a Borel injection from $Y$ into $X$. Here a Borel injection is merely an injective Borel map. The proof is a repetition of that of the classical Cantor-Bernstein theorem. However, we should remark that the reason it runs smoothly in this context is because of the important theorem of Luzin-Suslin that a Borel injection from a Polish space to another preserves Borelness of subsets.

In our context the following definition is needed. A Borel injection $f: X$ $\rightarrow Y$ is called a Borel embedding if the image of a Borel set under $f$ is Borel. Now the proof of the classical Cantor-Bernstein theorem can be repeated to show that if there exist Borel embeddings $f: X \rightarrow Y$ and $g: Y \rightarrow X$, then $X$ and $Y$ are Borel isomorphic. We also adopt the notation $f: X \hookrightarrow_{\mathrm{B}} Y$ to indicate that $f$ is a Borel embedding from $X$ into $Y$, and write $X \hookrightarrow_{\mathrm{B}} Y$, or simply $X \hookrightarrow Y$ if there is no danger of confusion, if there exists $f: X \hookrightarrow_{\mathrm{B}} Y$.

The following simple observations on Borel isomorphism and embeddability of ordinals will be useful. Let $\alpha<\beta$ be ordinals. Note that the canonical injection (namely the identity map) from $\alpha$ into $\beta$ is a Borel embedding (in fact a homeomorphic one). It follows that for $\alpha<\beta$ we have $\alpha \cong_{\mathrm{B}} \beta$ iff $\beta \hookrightarrow \alpha$. The following lemma is our main tool to show that $\beta$ Borel embeds into $\alpha<\beta$.

Lemma 2.9. Let $\alpha<\beta$ be ordinals, let $\left\{U_{i}\right\}_{i \in I}$ and $\left\{V_{j}\right\}_{j \in J}$ be pairwise disjoint open sets in $\alpha$ and $\beta$ respectively, and let $C$ and $D$ be the closed subsets $\alpha-\bigcup_{i \in I} U_{i}$ and $\beta-\bigcup_{j \in J} V_{j}$ of $\alpha$ and $\beta$ respectively. Suppose that there exist $k \in I, \psi: D \hookrightarrow_{\mathrm{B}} U_{k}$, and an injection $\pi: J \rightarrow I-\{k\}$ such that for every $j \in J$ there is an $\psi_{j}: V_{j} \hookrightarrow_{\mathrm{B}} U_{\pi(j)}$. Then $\beta$ Borel embeds into $\alpha-C$, thus into $\alpha$, and $\beta \cong_{\mathrm{B}} \alpha$.

Proof. Let $\phi: \beta \rightarrow \alpha-C$ be the piecewise defined map from $\psi$ and the $\psi_{j}$ 's. Clearly, $\phi$ is injective. If $B \subseteq \beta$ is Borel, then $B \cap D$ is Borel in $D$ and $B \cap V_{j}$ is Borel for each $j \in J$. Hence, $\phi$ " $B$ is Borel in each $U_{j}$. By the gluing lemma, $\phi " B$ is Borel in $\alpha$, and from this it follows that $B$ is also Borel in 
$\alpha-C$. Similarly, if $B \subseteq \alpha-C$ is Borel, then $\phi^{-1}\left(B \cap U_{k}\right)$ is Borel in $D$ and for any $l \in J-\{k\}, \phi^{-1}\left(B \cap U_{l}\right)$ is Borel in $V_{\pi^{-1}(l)}$, hence $\phi^{-1} B$ is Borel in $\beta$ again by the gluing lemma.

Under the hypothesis of the above lemma a particularly easy way to guarantee $D \hookrightarrow U_{k}$ for some $k$ is to make sure that ot $(D) \leq \operatorname{ot}\left(U_{k}\right)$. Note that the lemma is still meaningful even when $\alpha=\beta$. Specifically, if $\alpha \geq \omega$ and $C \subseteq \kappa \cdot \alpha$ is closed with order type $\leq \kappa$, then the lemma implies that $\kappa \cdot \alpha-C \cong_{\mathrm{B}} \kappa \cdot \alpha$. We record this observation for later use.

Corollary 2.10. If $\alpha \geq \omega$ and $C \subseteq \kappa \cdot \alpha$ is closed with order type $\leq \kappa$, then $\kappa \cdot \alpha-C \cong \cong_{\mathrm{B}} \kappa \cdot \alpha$.

3. The classification. In this section we classify all ordinals up to Borel isomorphism. Since all countable ordinals are Borel isomorphic, and $\alpha \neq_{\mathrm{B}} \beta$ whenever $|\alpha| \neq|\beta|$, we can restrict ourselves to ordinals $\alpha$ and $\beta$ so that $\kappa \leq \alpha<\beta<\kappa^{+}$for some uncountable cardinal $\kappa$. As remarked before, in order to show that $\alpha \cong_{\mathrm{B}} \beta$, it suffices to find a Borel embedding of $\beta$ into $\alpha$.

We split the proof of the classification Theorem 1.1 into three parts. First, we show that all $\beta \geq \kappa \cdot \operatorname{cof}(\kappa)$ are Borel isomorphic to $\kappa \cdot \operatorname{cof}(\kappa)$. Second, we show that for singular $\kappa, \kappa \cdot \operatorname{cof}(\kappa)$ is Borel isomorphic to $\kappa$. Finally, we identify the Borel isomorphism types between $\kappa$ and $\kappa^{2}$ for regular $\kappa$.

For the first part, we need the following lemma.

Lemma 3.1. If $\omega \leq \alpha \leq \kappa$, then $\kappa \cdot \alpha^{2} \cong_{\mathrm{B}} \kappa \cdot \alpha$.

Proof. We first show $\kappa \cdot \alpha^{2} \hookrightarrow \kappa \cdot \alpha \cdot 2$. Let $C=\left\{\kappa \cdot \xi: \xi<\alpha^{2}\right\}$. Then $C$ is a club in $\kappa \cdot \alpha^{2}$ and $\kappa \cdot \alpha^{2}-C$ consists of $\left|\alpha^{2}\right|=|\alpha|$ many maximal disjoint open intervals each of which is a copy of the ordinal $\kappa$. We refer to these maximal open intervals as $\kappa$-blocks.

For $\kappa \cdot \alpha \cdot 2$ we let $D=\{\kappa \cdot \alpha+\kappa \cdot \xi: \xi<\alpha\}$. Then $\kappa \cdot \alpha \cdot 2-D$ consists of a copy of $\kappa \cdot \alpha$ and $|\alpha|$ many $\kappa$-blocks. Now since ot $(C) \leq \kappa \cdot \alpha, C$ can be Borel embedded into the copy of $\kappa \cdot \alpha$. Since there are the same number of $\kappa$-blocks in the remaining parts of the two ordinals, they can be paired off. Lemma 2.9 gives the desired Borel embedding.

Second, we show $(\kappa \cdot \alpha) \cdot 2 \hookrightarrow \kappa \cdot \alpha$. Let $C_{1}=\{\kappa \cdot \xi: \xi<\alpha\}$ and let $C_{2}=\{\kappa \cdot \alpha+\kappa \cdot \xi: \xi<\alpha\}$. Since ot $\left(C_{1}\right)=\operatorname{ot}\left(C_{2}\right)=\alpha \leq \kappa$, we can embed $C_{1}$ into the first $\kappa$-block of $\kappa \cdot \alpha$, and $C_{2}$ into the second $\kappa$-block of $\kappa \cdot \alpha$. Now we are in a position to apply Lemma 2.9 again, since there are again the same number $|\alpha \cdot 2|=|\alpha|$ of $\kappa$-blocks in the remaining part of the two ordinals.

Theorem 3.2. If $\kappa \cdot \operatorname{cof}(\kappa) \leq \alpha<\kappa^{+}$, then $\alpha \cong_{\mathrm{B}} \kappa \cdot \operatorname{cof}(\kappa)$. 
Proof. We prove by induction that $\alpha$ can be partitioned into countably many Borel subsets $A_{0}, A_{1}, \ldots$ such that each $A_{n}$ embeds into $\kappa \cdot \operatorname{cof}(\kappa)$. This gives a Borel embedding of $\alpha$ into $\kappa \cdot \operatorname{cof}(\kappa) \cdot \omega$, which embeds into $\kappa \cdot \operatorname{cof}(\kappa)^{2}$ and hence in $\kappa \cdot \operatorname{cof}(\kappa)$ by the preceding lemma.

The statement is certainly true for $\alpha=\kappa \cdot \operatorname{cof}(\kappa)$. The successor case is also easy. We assume $\alpha$ is a limit ordinal. Let $C=\left\{x_{\beta}: \beta<\operatorname{cof}(\alpha)\right\}$ be a club in $\alpha$, with $x_{0}=0$. Since $\operatorname{cof}(\alpha) \leq \kappa$ (because $\left.\alpha<\kappa^{+}\right), C$ can be embedded into $\kappa$ and thus in $\kappa \cdot \operatorname{cof}(\kappa)$. For each $\beta<\operatorname{cof}(\alpha)$ let $I_{\beta}=\left(x_{\beta}, x_{\beta+1}\right)$. The $I_{\beta}$ 's are pairwise disjoint open subsets of $\alpha$ such that $\alpha-C=\bigcup_{\beta<\operatorname{cof}(\alpha)} I_{\beta}$. Also for each $\beta<\operatorname{cof}(\alpha), I_{\beta}$ is a copy of an ordinal $<\alpha$. Thus by the inductive hypothesis, or because the order type of $I_{\beta}$ is $<\kappa \cdot \operatorname{cof}(\kappa)$, for every $\beta<\operatorname{cof}(\alpha)$ there is a pairwise disjoint family $\left\{A_{\beta, n}^{\prime}: n \in \mathbb{N}\right\}$ such that $I_{\beta}=\bigcup_{n<\omega} A_{\beta, n}^{\prime}$, every $A_{\beta, n}^{\prime}$ is Borel in $I_{\beta}$, and there is a Borel embedding $\varphi_{\beta, n}: A_{\beta, n}^{\prime} \hookrightarrow \mathrm{B} \kappa \cdot \operatorname{cof}(\kappa)$.

Define $A_{n}^{\prime}:=\bigcup_{\beta<\operatorname{cof}(\alpha)} A_{\beta, n}^{\prime}$. Since each $A_{n}^{\prime} \cap I_{\beta}=A_{\beta, n}^{\prime}$ is Borel in $I_{\beta}$, it follows that $A_{n}^{\prime}$ is Borel in $\alpha$ by the gluing lemma. Also for every $n<\omega$, $A_{n}^{\prime}=\bigcup_{\beta<\operatorname{cof}(\alpha)} A_{\beta, n}^{\prime}$ is Borel embeddable in $\kappa \cdot \operatorname{cof}(\kappa) \cdot \operatorname{cof}(\kappa)$, and thus $A_{n}^{\prime}$ embeds into $\kappa \cdot \operatorname{cof}(\kappa)$ by the preceding lemma. Then $A_{0}=C, A_{n+1}=A_{n}^{\prime}$ is the required decomposition of $\alpha$.

Thus between any cardinal $\kappa$ and its successor $\kappa^{+}$there are no new isomorphism types after $\kappa \cdot \operatorname{cof}(\kappa)$. For singular $\kappa$, there is in fact only one isomorphism type after all.

TheOREM 3.3. If $\kappa$ is singular and $\kappa \leq \alpha<\kappa^{+}$, then $\alpha \cong_{\mathrm{B}} \kappa$.

Proof. In view of Theorem 3.2 it suffices to prove that $\kappa \cdot \operatorname{cof}(\kappa) \cong_{\mathrm{B}} \kappa$. Fix a club-in- $\kappa$ sequence $\left\langle\lambda_{\zeta}: \zeta<\operatorname{cof}(\kappa)\right\rangle$ of cardinals such that $\operatorname{cof}(\kappa)<\lambda_{\zeta}<\kappa$. Let

$$
C=\{\kappa \cdot \xi: \xi<\operatorname{cof}(\kappa)\} \cup \bigcup_{\xi<\operatorname{cof}(\kappa)}\left\{\kappa \cdot \xi+\lambda_{\zeta}: \zeta<\operatorname{cof}(\kappa)\right\} .
$$

This is a club in $\kappa \cdot \operatorname{cof}(\kappa)$ of order type $\operatorname{cof}(\kappa)^{2}$. Again $\kappa \cdot \operatorname{cof}(\kappa)-C$ can be written as a union of $\left|\operatorname{cof}(\kappa)^{2}\right|=\operatorname{cof}(\kappa)$ many maximal disjoint open intervals, or blocks, each of which is a copy of some $\lambda_{\zeta}$. Moreover, for each $\zeta<\operatorname{cof}(\kappa)$ there are exactly $\operatorname{cof}(\kappa)$ many $\lambda_{\zeta \text {-blocks. }}$

On the other hand, $D=\left\{\lambda_{\zeta}: \zeta<\operatorname{cof}(\kappa)\right\}$ is a club in $\kappa$ of order type $\operatorname{cof}(\kappa)$, and $\kappa-D$ is the union of $\operatorname{cof}(\kappa)$ many blocks each of which is a copy of some $\lambda_{\zeta}$. However, for each $\zeta<\operatorname{cof}(\kappa)$ there is exactly one $\lambda_{\zeta}$-block in $\kappa-D$, which we denote by $B_{\zeta}$.

We now define a Borel embedding from $\kappa \cdot \operatorname{cof}(\kappa)$ into $\kappa$ in view of Lemma 2.9. First note that $C$ embeds into $B_{0}$ since $\lambda_{0} \geq \operatorname{cof}(\kappa)^{+}>\operatorname{cof}(\kappa)^{2}$. Then for each $\zeta<\operatorname{cof}(\kappa)$ we let all $\operatorname{cof}(\kappa)$ many $\lambda_{\zeta}$-blocks in $\kappa \cdot \operatorname{cof}(\kappa)$ embed 
into the $\lambda_{\zeta+1}$-block $B_{\lambda_{\zeta+1}}$ of $\kappa$. This is possible since $\lambda_{\zeta+1}>\left|\lambda_{\zeta} \cdot \operatorname{cof}(\kappa)\right|$ $=\lambda_{\zeta}$.

Finally, we consider ordinals between $\kappa$ and $\kappa^{2}$ when $\kappa$ is a regular uncountable cardinal. Any such ordinal can be written as $\kappa \cdot \alpha+\beta$ with $0<\alpha \leq \kappa$ and $0 \leq \beta<\kappa$.

Lemma 3.4. If $0<\alpha \leq \kappa$ and $0 \leq \beta<\kappa$, then $\kappa \cdot \alpha+\beta \cong_{\mathrm{B}} \kappa \cdot \alpha$.

Proof. This is immediate when $\beta$ is finite, so assume $\beta$ is infinite. In this case $\kappa \cdot \alpha+\beta=\kappa \cdot \alpha+1+\beta$ is the disjoint union of the open sets $[0, \kappa \cdot \alpha+1)$ and $(\kappa \cdot \alpha, \kappa \cdot \alpha+\beta)$. In other words, $\kappa \cdot \alpha+\beta$ is homeomorphic to the direct sum $(\kappa \cdot \alpha+1) \oplus \beta$. Replacing $\beta$ with the Borel isomorphic $\beta+1$, we are allowed to transpose the disjoint open parts:

$$
(\kappa \cdot \alpha+1) \oplus \beta \cong_{\mathrm{B}}(\kappa \cdot \alpha+1) \oplus(\beta+1) \cong(\beta+1) \oplus(\kappa \cdot \alpha+1) .
$$

Finally, $(\beta+1) \oplus(\kappa \cdot \alpha+1) \cong \beta+1+\kappa \cdot \alpha+1=\kappa \cdot \alpha+1 \cong_{\mathrm{B}} \kappa \cdot \alpha$.

We can therefore restrict our attention to ordinals of the form $\kappa \cdot \alpha$ for $0<$ $\alpha \leq \kappa$. It follows immediately from Lemma 2.9 that $\kappa \cdot \alpha \cong_{\mathrm{B}} \kappa \cdot \beta$ whenever $|\alpha|=|\beta|$. To motivate the converse, suppose towards a contradiction that $\theta$ is a Borel isomorphism between $\omega_{1} \cdot 2$ and $\omega_{1}$. The larger ordinal $\omega_{1} \cdot 2$ consists of two copies $B_{1}, B_{2}$ of $\omega_{1}$ (and a limit point), while the smaller ordinal $\omega_{1}$ has only one block. Each of the copies is Borel in $\omega_{1} \cdot 2$ and therefore so are their images $\theta$ " $B_{1}$ and $\theta^{\prime \prime} B_{2}$. By Lemma 2.3 , both images either contain or omit a club. Since $\theta^{\prime \prime} B_{1}$ and $\theta^{\text {" }} B_{2}$ are disjoint, and any two clubs meet, one of the images, say $\theta^{\prime \prime} B_{1}$, must omit a club $C$. This closed set splits $\omega_{1}$ into open blocks. One can construct a stationary and costationary $S \subseteq B_{1}$ such that $\theta$ " $S$ contains at most one point in each block. Hence, $\theta$ " $S$ is Borel in $\omega_{1}$ by the gluing lemma, but $S$ is not Borel in $B_{1}$ and hence not in $\omega_{1} \cdot 2$, a contradiction. The argument in the proof of the following theorem is a generalization of this idea.

THEOREM 3.5. Let $\kappa$ be a regular uncountable cardinal and let $\alpha<\beta$ $\leq \kappa$. If $|\alpha| \neq|\beta|$, then $\kappa \cdot \alpha \neq_{\mathrm{B}} \kappa \cdot \beta$.

Proof. We may assume without loss of generality that $|\alpha|<|\beta|$. Let $C_{0}=$ $\{\kappa \cdot \xi: \xi<\alpha\}$ and $D_{0}=\{\kappa \cdot \xi: \xi<\beta\}$. By Corollary $2.10, \kappa \cdot \alpha \cong_{\mathrm{B}} \kappa \cdot \alpha-C_{0}$ and $\kappa \cdot \beta \cong_{\mathrm{B}} \kappa \cdot \beta-D_{0}$. Thus it suffices to show that $\kappa \cdot \alpha-C_{0} \approx_{\mathrm{B}} \kappa \cdot \beta-D_{0}$.

Toward a contradiction we assume that $\theta: \kappa \cdot \beta-D_{0} \rightarrow \kappa \cdot \alpha-C_{0}$ is a Borel isomorphism. As before $\kappa \cdot \alpha-C_{0}$ consists of $|\alpha|$ many $\kappa$-blocks, which we denote in increasing order by $A_{\zeta}$ for $\zeta<\alpha$. Similarly $\kappa \cdot \beta-D_{0}$ consists of $|\beta|$ many $\kappa$-blocks, which we denote in increasing order by $B_{\xi}$ for $\xi<\beta$.

Claim 1. There is a $\xi<\beta$ such that for every $\zeta<\alpha, A_{\zeta} \cap \theta^{\text {" }} B_{\xi}$ is nonstationary in $A_{\zeta}$. 
Proof. Note that the $\kappa$-blocks $A_{\zeta}, B_{\xi}$ are open. For every $\xi<\beta, \theta$ " $B_{\xi}$ is Borel in $\kappa \cdot \alpha$, thus for every $\zeta<\alpha, A_{\zeta} \cap \theta^{\text {" }} B_{\xi}$ is Borel in $\kappa \cdot \alpha$ and thus in $A_{\zeta}$. But $A_{\zeta}$ is a copy of the regular $\kappa$, hence $A_{\zeta} \cap \theta$ " $B_{\xi}$ must either contain or miss a club in $A_{\zeta}$ by Lemma 2.3. Since two clubs necessarily meet, for every $\zeta<\alpha$ there can be at most one $\xi<\beta$ such that $A_{\zeta} \cap \theta^{\text {" }} B_{\xi}$ contains a club in $A_{\zeta}$. Because $|\alpha|<|\beta|$, there must be a $\xi<\beta$ such that for every $\zeta<\alpha$, $A_{\zeta} \cap \theta$ " $B_{\xi}$ is nonstationary in $A_{\zeta}$.

Fix $\xi$ satisfying Claim 1.

Claim 2. There is a stationary $S \subseteq B_{\xi}$ such that $\theta$ " $S \subseteq A_{\zeta}$ for some $\zeta<\alpha$.

Proof. For each $\zeta<\alpha$ let $B_{\xi, \zeta}=B_{\xi} \cap \theta^{-1}\left(A_{\zeta}\right)$. Then $B_{\xi}=\bigcup_{\zeta<\alpha} B_{\xi, \zeta}$. Since $B_{\xi}$ is a copy of $\kappa$ and $\alpha<\kappa$, it follows from the regularity of $\kappa$ that $B_{\xi}$ is not the union of $|\alpha|$ many nonstationary sets. Hence, there must be a $\zeta<\alpha$ such that $B_{\xi, \zeta}$ is stationary. This stationary set $S=B_{\xi, \zeta}$ has the required property.

We now have a stationary set $S \subseteq B_{\xi}$ such that $\theta$ " $S$ is entirely contained in $A_{\zeta}$. Since $\theta$ " $B_{\xi}$ is nonstationary on every $\kappa$-block of $\kappa \cdot \alpha, \theta$ " $S$ is nonstationary in $A_{\zeta}$. Note that both $B_{\xi}$ and $A_{\zeta}$ are copies of the regular cardinal $\kappa$.

Let $C$ be a club in $A_{\zeta}$ such that $\theta$ " $S \cap C=\emptyset$. Then $A_{\zeta}-C$ can be written as the disjoint union of maximal open intervals, say $A_{\zeta}-C=\bigcup_{i \in \kappa} U_{i}=$ $\bigcup_{i \in \kappa}\left(\gamma_{i}, \gamma_{i+1}\right)$. Note that $\theta$ " $S \subseteq \bigcup_{i \in \kappa} U_{i}$.

Claim 3. There is an $S_{1} \subseteq S$ which is stationary and costationary in $B_{\xi}$ such that $\theta$ " $S_{1} \cap U_{i}$ is Borel in $U_{i}$ for every $i \in \kappa$.

Proof. For any $x \in S$, denote by $\operatorname{block}(x) \in \kappa$ the index of the block that $\theta(x)$ is in, that is, $\theta(x) \in U_{\text {block }(x)}$. We will construct a club $D$ such that for $x, y \in D \cap S$ with $x \neq y, \operatorname{block}(x) \neq \operatorname{block}(y)$. Then $S_{0}:=D \cap S$ is a stationary set such that $\mid \theta$ " $S_{0} \cap U_{i} \mid \leq 1$. This trivially implies that $\theta$ " $S_{0} \cap U_{i}$ is Borel in $U_{i}$ for every $i \in \kappa$. Furthermore, let $S_{1} \subseteq S_{0}$ be any stationary and costationary subset. Then $\theta$ " $S_{1} \cap U_{i}$ is Borel in $U_{i}$ for every $i \in \kappa$.

To construct this club $D$, we define a function $g: B_{\xi} \rightarrow B_{\xi}$ and then let $D$ be the set of closure points of $g$, that is, $D=\left\{\alpha \in B_{\xi}: \forall \beta<\alpha(g(\beta)<\alpha)\right\}$. Let $x \in B_{\xi}$ be arbitrary. Let $B=\{\operatorname{block}(z): z \in S \wedge z \leq x\}$. Since $\kappa$ is regular, $B$ is bounded in $\kappa$. Let $g(x)=\sup \left\{x^{\prime} \in B_{\xi} \cap S: \operatorname{block}\left(x^{\prime}\right) \in B\right\}$. Since $\kappa$ is regular and $\theta$ is one-to-one, $g(x) \in B_{\xi}$. To see this works, suppose $x, y \in S_{0}=D \cap S$ with $x<y$. Since $y \in D, g(x)<y$. Thus, $\operatorname{block}(y) \notin$ $\{\operatorname{block}(z): z \leq x \wedge z \in S\}$, a set which includes $\operatorname{block}(x)$.

Since $\theta$ " $S_{1} \cap U_{i}$ is Borel in $U_{i}$ for every $i<\kappa, \theta$ " $S_{1}$ is Borel in $\bigcup_{i<\kappa} U_{i}$ by the gluing lemma, and hence $\theta$ " $S_{1}$ is Borel in $A_{\zeta}$ and also in $\kappa \cdot \alpha$. But $S_{1}$ is 
not Borel in $B_{\xi}$ by Lemma 2.3 and thus not Borel in $\kappa \cdot \beta$. This contradicts the assumption that $\theta$ is a Borel embedding.

This completes the proof of Theorem 1.1: if $\kappa$ is singular or countable, all ordinals between $\kappa$ and $\kappa^{+}$are Borel isomorphic by Theorems 3.2 and 3.3 , and if $\kappa$ is regular and uncountable, the Borel isomorphism types are precisely $\kappa \cdot \lambda$ for cardinals $1 \leq \lambda \leq \kappa$ by Theorems 3.2 and 3.5.

4. The situation assuming determinacy. In this section we consider the Borel isomorphism question assuming now ZF + AD. The results of $\S 3$ were proved assuming $A C$, and no longer hold in this context. We first consider the question of which sets of ordinals are Borel. Under AD, the club filter on $\omega_{1}$ is a measure, that is, there are no stationary, costationary subsets of $\omega_{1}$ (which would be non-Borel sets). This suggests the possibility of the following theorem. This theorem can be proved either using the "simple set" type analysis occurring in the analysis of measures on $\omega_{1}$, or by an indiscernibility argument. We give the proof following the indiscernibility argument. We will use the basic theory of the Silver indiscernibles. The reader can consult $\S 30$ of [4] or $\S 8 \mathrm{H}$ of [7] for a presentation of this theory. The measure analysis can be found in [2] or [3] (we make a few comments at the end about how the following proof can be modified along those lines).

THEOREM 4.1 (ZF+AD). Every subset of $\omega_{1}$ is Borel, and can be written in the form $\bigcup_{n}\left(F_{n} \cap U_{n}\right)$ where $F_{n}$ is closed and $U_{n}$ is open.

Proof. Let $A \subseteq \omega_{1}$. The only AD fact we use is that $A \in L[x]$ for some real $x$. Let $C \subseteq \omega_{1}$ be the canonical set of Silver indiscernibles for $L[x]$, so $C$ is club in $\omega_{1}$. Let $C^{\prime}$ denote the set of limit points of $C$, so $C^{\prime}$ is also a club set of indiscernibles for $L[x]$.

There are finitely many indiscernibles $\alpha_{0}<\cdots<\alpha_{a}<\omega_{1}$, and finitely many $\omega_{1}, \ldots, \omega_{b}$ and a term $u$ such that $A=u^{L[x]}\left(\alpha_{0}, \ldots, \alpha_{a}, \omega_{1}, \ldots, \omega_{b}\right)$. For notational simplicity we suppress the $\omega_{1}, \ldots, \omega_{b}$ as well as the superscript $L[x]$, and just write $A=u(\vec{\alpha})$.

By the type of an ordinal $\alpha<\omega_{1}$ we mean the specification of:

(1) finitely many $\beta_{1}, \ldots, \beta_{c} \leq \alpha_{a}$ in $C^{\prime}$,

(2) an $L[x]$ term $t=t^{L[x]}\left(x_{1}, \ldots, x_{c}, y_{1}, \ldots, y_{n}\right)$ for some $n \in \omega$.

There are clearly only countably many types; let us enumerate them as $T_{1}, T_{2}, \ldots$ For a type $T$ as above, we say an ordinal $\alpha<\omega_{1}$ has type $T$ provided $\alpha=t^{L[x]}\left(\vec{\beta}, \gamma_{1}, \ldots, \gamma_{n}\right)$ for some indiscernibles $\gamma_{1}<\cdots<\gamma_{n}<\omega_{1}$ in $C$ with $\gamma_{1}>\max (\vec{\alpha})$. Every countable ordinal is represented by some type, since $C$ is a generating set of indiscernible for $L[x]$.

For a type $T$ as above, we say $T$ is normal if the following statements are in $x^{\#}$ : 
(a) $t\left(\vec{\beta}, \gamma_{1}, \ldots, \gamma_{n}\right) \geq \gamma_{n}$.

(b) $t\left(\vec{\beta}, \gamma_{1}, \ldots, \gamma_{i}, \ldots, \gamma_{n}\right) \neq t\left(\vec{\beta}, \gamma_{1}, \ldots, \gamma_{i}^{\prime}, \ldots, \gamma_{n}\right)$ for $\gamma_{1}<\cdots<\gamma_{i}<$ $\gamma_{i}^{\prime}<\gamma_{i+1}<\cdots<\gamma_{n}$.

Every countable ordinal is represented by a normal type. This follows from two observations. First, if $t\left(\vec{\beta}, \gamma_{1}, \ldots, \gamma_{n}\right)<\gamma_{n}$ is in $x^{\#}$ then there is a term $u=u\left(\vec{x}, y_{1}, \ldots, y_{n-1}\right)$ such that $t\left(\vec{\beta}, \gamma_{1}, \ldots, \gamma_{n}\right)=u\left(\vec{\beta}, \gamma_{1}, \ldots, \gamma_{n-1}\right)$ for all indiscernibles $\gamma_{1}<\cdots<\gamma_{n}$ (we again suppress the dependence on $\left.\omega_{1}, \ldots, \omega_{l}\right)$. Secondly, if $t\left(\vec{\beta}, \gamma_{1}, \ldots, \gamma_{i}, \ldots, \gamma_{n}\right)=t\left(\vec{\beta}, \gamma_{1}, \ldots, \gamma_{i}^{\prime}, \ldots, \gamma_{n}\right)$ is in $x^{\#}$, then for some term $t^{\prime}=t^{\prime}\left(\vec{x}, y_{1}, \ldots, y_{i-1}, y_{i+1}, \ldots, y_{n}\right)$ we have

$$
t\left(\vec{\beta}, \gamma_{1}, \ldots, \gamma_{n}\right)=t^{\prime}\left(\vec{\beta}, \gamma_{1}, \ldots, \gamma_{i-1}, \gamma_{i+1}, \ldots, \gamma_{n}\right) .
$$

Thus, we may eliminate variables until (b) is satisfied. From (b), note that we must actually have $t\left(\vec{\beta}, \gamma_{1}, \ldots, \gamma_{n}\right)>\gamma_{n}$ unless $n=1$ and $t\left(\vec{\beta}, \gamma_{1}\right)=\gamma_{1}$. We henceforth assume that all the $T_{n}$ are normal.

To show $A$ is Borel, it suffices to show that $A \cap X_{n}$ is Borel, where $X_{n}$ is the set of countable ordinals of type $T_{n}$. We also show that $A \cap X_{n}$ is of the form $\bigcup_{n}\left(F_{n} \cap U_{n}\right)$ as required. Henceforth, fix a type $T$ corresponding to a term $t=t\left(\vec{x}, y_{1}, \ldots, y_{n}\right)$, and we show $A \cap X$ is Borel ( $X$ being the set of ordinals of type $T$ ). By indiscernibility, we either have $X \subseteq A$ or $X \subseteq \omega_{1}-A$. So, either $A \cap X=X$ or $A \cap X=\emptyset$. It suffices therefore to show that $X$ is Borel, and in fact $X=\bigcup_{n}\left(F_{n} \cap U_{n}\right)$ for $F_{n}$ closed, $U_{n}$ open.

For the rest of the argument we suppress writing the fixed ordinals $\vec{\alpha}$ and $\vec{\beta}$, and consider only $X-(\max (\vec{\alpha})+1)$ (any countable set is clearly of the required form).

If $t\left(\gamma_{1}, \ldots, \gamma_{n}\right)=\gamma_{n}$, then $n=1$ and $X=C$, which is closed. So we may henceforth assume $t\left(\gamma_{1}, \ldots, \gamma_{n}\right)>\gamma_{n}$.

Consider first the simple case $n=1$. By indiscernibility, $t\left(\gamma_{1}\right)<\gamma_{2}$ for all indiscernibles $\gamma_{1}<\gamma_{2}$. So, there is exactly one element of $X$ between any indiscernible $\gamma \in C$ and the next indiscernible. Since $X \cap C=\emptyset$, it follows that $X$ is closed in $\omega_{1}-C$. Thus, $X$ is the intersection of a closed set (the closure of $X)$ and an open set $\left(\omega_{1}-C\right)$.

Consider now the general case $n>1$. By indiscernibility and wellfoundedness, $t(\vec{\gamma})$ is increasing in each argument. From $(\mathrm{b}), t(\vec{\gamma})$ is actually strictly increasing in each argument. We need the following rather standard claim.

ClaIm 4. There is permutation $\pi=\left(i_{1}, \ldots, i_{n}\right)$ of $\{1, \ldots, n\}$ with $i_{1}=n$ such that for all indiscernibles $\gamma_{1}<\cdots<\gamma_{n}, \delta_{1}<\cdots<\delta_{n}$, we have $t(\vec{\gamma})<t(\vec{\delta})$ iff

$$
\left(\gamma_{i_{1}}, \ldots, \gamma_{i_{n}}\right)<_{\text {lex }}\left(\delta_{i_{1}}, \ldots, \delta_{i_{n}}\right) .
$$

For $t$ and $\pi$ as in the claim, we say the term $t$ has type $\pi$. We adopt the practice of writing the arguments to $t$ in any order, which causes no confusion as we actually only evaluate $t\left(\gamma_{1}, \ldots, \gamma_{n}\right)$ for $\gamma_{1}<\cdots<\gamma_{n}$. For example, we 
may write $t\left(\gamma_{i_{1}}, \ldots, \gamma_{i_{n}}\right)$. Also, we say the sequence $\left(\gamma_{i_{1}}, \ldots, \gamma_{i_{n}}\right)$ is of type $\pi$ if it is order-isomorphic to $\pi=\left(i_{1}, \ldots, i_{n}\right)$.

We use the following technical result.

Claim 5. Let $t=t\left(y_{1}, \ldots, y_{n}\right)$ be an $L[x]$ term of type $\pi=\left(i_{1}, \ldots, i_{n}\right)$. Then one of the following holds:

(1) For every $\gamma_{1}<\cdots<\gamma_{n}$ in $C$ with $\gamma_{i_{n}} \in C^{\prime}$ we have

$$
t(\vec{\gamma})=\sup \left\{t\left(\gamma_{i_{1}}, \ldots, \gamma_{i_{n-1}}, \gamma^{\prime}\right): \gamma^{\prime}<\gamma_{i_{n}} \wedge \gamma^{\prime} \in C\right\}
$$

(2) For every $\gamma_{1}<\cdots<\gamma_{n}$ in $C$ with $\gamma_{i_{n}} \in C^{\prime}$ we have

$$
t(\vec{\gamma})>\sup \left\{t\left(\gamma_{i_{1}}, \ldots, \gamma_{i_{n-1}}, \gamma^{\prime}\right): \gamma^{\prime}<\gamma_{i_{n}} \wedge \gamma^{\prime} \in C\right\}
$$

Proof. First note that for all $\gamma_{1}<\cdots<\gamma_{n}$ in $C$ with $\gamma_{i_{n}} \in C^{\prime}$,

$$
\begin{aligned}
\sup \left\{t\left(\gamma_{i_{1}}, \ldots, \gamma_{i_{n-1}}, \gamma^{\prime}\right):\right. & \left.\gamma^{\prime}<\gamma_{i_{n}} \wedge \gamma^{\prime} \in C\right\} \\
=\sup \{ & t\left(\gamma_{i_{1}}, \ldots, \gamma_{i_{n-1}}, \gamma^{\prime}\right): \gamma^{\prime}<\gamma_{i_{n}} \\
& \left.\wedge t\left(\gamma_{i_{1}}, \ldots, \gamma_{i_{n-1}}, \gamma^{\prime}\right)<t\left(\gamma_{i_{1}}, \ldots, \gamma_{i_{n-1}}, \gamma_{i_{n}}\right)\right\}
\end{aligned}
$$

The set in the first supremum is contained in the set of the second supremum, so the first supremum is less than or equal to the second. For the other direction note that if $\gamma^{\prime}<\gamma_{i_{n}}$, then $\gamma^{\prime}=w(\vec{\delta})$ for some term $w$ and indiscernibles $\vec{\delta}$ below $\gamma_{i_{n}}$. So, if $t\left(\gamma_{i_{1}}, \ldots, \gamma^{\prime}\right)=t\left(\gamma_{i_{1}}, \ldots, w(\vec{\delta})\right)<t\left(\gamma_{i_{1}}, \ldots, \gamma_{i_{n}}\right)$, then by indiscernibility for large enough $\eta<\gamma_{i_{n}}$ in $C$ we have $t\left(\gamma_{i_{1}}, \ldots, \gamma^{\prime}\right) \leq$ $t\left(\gamma_{i_{1}}, \ldots, \gamma_{i_{n-1}}, \eta\right)$. Thus, the two suprema above are equal. It follows that there is an $L[x]$ term $v$ such that for all $\vec{\gamma}$ in $C$ with $\gamma_{i_{n}} \in C^{\prime}$ we have $v(\vec{\gamma})=\sup \left\{t\left(\gamma_{i_{1}}, \ldots, \gamma_{i_{n-1}}, \gamma^{\prime}\right): \gamma^{\prime}<\gamma_{i_{n}} \wedge \gamma^{\prime} \in C\right\}$. The claim then follows by indiscernibility.

If the first alternative in Claim 5 holds, then we say $t$ is of continuous type, and otherwise of discontinuous type.

We also require the following result.

Claim 6. Let $t\left(y_{1}, \ldots, y_{n}\right)$ be a term of type $\pi=\left(i_{1}, \ldots, i_{n}\right)$. Suppose $j<n$ and $\gamma_{1}<\cdots<\gamma_{n}$ are in $C$ with $\gamma_{i_{j}} \in C^{\prime}$. Then

$$
\begin{array}{r}
t(j)\left(\gamma_{i_{1}}, \ldots, \gamma_{i_{j}}\right):=\sup \left\{t\left(\gamma_{i_{1}}, \ldots, \gamma_{i_{j-1}}, \delta_{i_{j}}, \ldots, \delta_{i_{n}}\right): \delta_{i_{j}}<\gamma_{i_{j}},\right. \\
\left.\delta_{i_{j}}, \ldots, \delta_{i_{n}} \in C, \text { and }\left(\gamma_{i_{1}}, \ldots, \delta_{i_{n}}\right) \text { is of type } \pi\right\}
\end{array}
$$

is not in the range of $t\lceil C$ (that is, is not in $X$ ).

Proof. Note that as in Claim 5, the function $t(j)$ defined above is given by an $L[x]$ term $v\left(\gamma_{i_{1}}, \ldots, \gamma_{i_{j}}\right)$. Suppose that

$$
t(j)\left(\gamma_{i_{1}}, \ldots, \gamma_{i_{j}}\right)=v\left(\gamma_{i_{1}}, \ldots, \gamma_{i_{j}}\right)=t\left(\delta_{i_{1}}, \ldots, \delta_{i_{n}}\right),
$$

where all the ordinals are in $C$. By indiscernibility, we may move the ordinals so that all of them lie in $C^{\prime}$, and this equation is still satisfied. Since $j<n$, there is some $\delta_{i_{k}}$ which is not equal to any of the $\gamma_{i_{1}}, \ldots, \gamma_{i_{j}}$. Since all 
the ordinals are in $C^{\prime}$, we can move $\delta_{i_{k}}$ to a new value $\delta_{i_{k}}^{\prime} \in C$ keeping the same relative ordering of the ordinals. By indiscernibility the equation still holds, which contradicts the fact that $t$ is strictly increasing in each argument.

We now show that $X$ is the intersection of a closed and an open set in $\omega_{1}$. If we are in case (1) of Claim 5 , then $\bar{X}-X=\bigcup_{j<n} A_{j}$ where $A_{j}$ is the set of ordinals of the form $t(j)\left(\gamma_{i_{1}}, \ldots, \gamma_{i_{j}}\right)$, where all of the ordinals are in $C$, and $\gamma_{i_{j}} \in C^{\prime}$. To see this, suppose $\eta \in \bar{X}-X$. Then $\eta$ is the increasing limit of a sequence $\eta_{n}=t\left(\gamma_{i_{1}}^{n}, \ldots, \gamma_{i_{n}}^{n}\right)$. Let $j$ be least such that $\left\{\gamma_{i_{j}}^{n}\right\}_{m \in \omega}$ is not eventually constant. For $k<j$, let $\gamma_{i_{k}}$ be the eventual value of the $\gamma_{i_{k}}^{n}$, and let $\gamma_{i_{j}}=\sup _{n} \gamma_{i_{j}}^{n}$. Thus, $\gamma_{i_{j}} \in C^{\prime}$. Since $t$ is of type $\pi$ it follows from the definition of $t(j)$ that $\eta=t(j)\left(\gamma_{i_{1}}, \ldots, \gamma_{i_{j}}\right)$. If $j=n$, then from case (1) we have $t(n)\left(\gamma_{i_{1}}, \ldots, \gamma_{i_{n}}\right)=\sup _{\gamma^{\prime}<\gamma_{i_{n}}} t\left(\gamma_{i_{1}}, \ldots, \gamma_{i_{n}}\right)=t\left(\gamma_{i_{1}}, \ldots, \gamma_{i_{n}}\right) \in X$, a contradiction. So, $\bar{X}-X \subseteq \bigcup_{j<n} A_{j}$. The reverse inclusion follows from Claim 6. Thus, $X=\bar{X}-F$, where $F=\bigcup_{j<n} A_{j}$. Finally, note that $\bigcup_{j<n} A_{j}$ is closed by a similar argument. If we are in case (2) of Claim 5, the argument is similar, except we have $\bar{X}-X=\bigcup_{j \leq n} A_{j}$.

As we mentioned above, the above proof can be given using the measure analysis on $\omega_{1}$. Say that a set $A \subseteq \omega_{1}$ is very simple if there is a club $C \subseteq \omega_{1}$, an $n \in \omega$, and an $h: C^{n} \rightarrow \omega_{1}$ with $A=h^{*}\left(C^{n}\right)$ where $h$ satisfies:

(1) $h \uparrow C^{n}$ is strictly increasing in each argument.

(2) There is a permutation $\pi=\left(i_{1}, \ldots, i_{n}\right)$ of $\left\{1, \ldots, i_{n}\right\}$ with $i_{1}=n$ such that for all $\alpha_{1}<\cdots<\alpha_{n}$ in $C$ and all $\beta_{1}<\cdots<\beta_{n}$ in $C$, $h(\vec{\alpha})<h(\vec{\beta})$ iff $\left(\alpha_{i_{1}}, \ldots, \alpha_{i_{n}}\right)<_{\operatorname{lex}}\left(\beta_{i_{1}}, \ldots, \beta_{i_{n}}\right)$.

(3) The conclusion of Claim 5 holds, using the club set $C$ instead of the set of indiscernibles and $h$ in place of $t$.

(4) The conclusion of Claim 6 holds, again using the current club set $C$ and $h$.

The analysis of measures on $\omega_{1}$ (cf. [2] or [3]) shows that for any measure $\nu$ on $\omega_{1}$, there is an $n \in \omega$ and an $h: \omega_{1}^{n} \rightarrow \omega_{1}$ such that for any $A \subseteq \omega_{1}$, $\nu(A)=1$ iff there is a club $C \subseteq \omega_{1}$ such that $h^{\prime \prime}\left(C^{n}\right) \subseteq A$. However, for any function $h: \omega_{1}^{n} \rightarrow \omega_{1}$, straightforward partition arguments show that there is an $n^{\prime} \leq n$ such that $h$ only depends on a subset of its arguments of size $n^{\prime}$, and that as a function of $n^{\prime}$ arguments it satisfies the above four properties on a club set. Thus, for every measure $\nu$ on $\omega_{1}$, there is a very simple set $A$ such that $\nu(A)=1$. Given this, the usual argument (due to Kunen) using the fact that every countably additive ideal on a $\lambda<\Theta$ can be extended to a measure (i.e., every set in the ideal has measure zero) shows that every subset of $\omega_{1}$ is a countable union of very simple sets. The proof of Theorem 4.1 now shows that every very simple set is an intersection of a 
closed and an open set (the four properties above were all that was used in this argument).

Theorem 4.1 can be pushed a little higher, which we do in the next theorem. We can again either prove this result using the measure analysis or using indiscernibles; we again give the proof using indiscernibles. The proof is a slight extension of the proof of Theorem 4.1.

ThEOREM $4.2(\mathrm{ZF}+\mathrm{AD})$. For every $\lambda<\omega_{2}$, every subset of $\lambda$ is Borel and can be written as $\bigcup_{n}\left(F_{n} \cap U_{n}\right)$ where $F_{n}$ is closed and $U_{n}$ is open.

Proof. Fix $\lambda<\omega_{2}$ and $A \subseteq \lambda$. Let $\prec$ be a wellordering of $\omega_{1}$ of length $\lambda$. Fix a real $x$ such that $\prec$ lies in $L[x]$. We may write $\prec=u\left(\alpha_{1}, \ldots, \alpha_{a}\right.$, $\left.\omega_{1}, \ldots, \omega_{b}\right)$ for some $\alpha_{1}<\cdots<\alpha_{a}$ in $C$ (the set of indiscernibles for $L[x]$ ) and some $L[x]$ term $u$. Let $A^{\prime}=\left\{\gamma<\omega_{1}:|\gamma|_{\prec} \in A\right\}$. Increasing the set $\vec{\alpha}$ and the value of $b$ if necessary, we may assume that $A^{\prime}$ is definable from $\vec{\alpha}$ and $\omega_{1}, \ldots, \omega_{b}$. Thus, $A=w\left(\vec{\alpha}, \omega_{1}, \ldots, \omega_{b}\right)$ for some term $w$.

We have the notions of type and normal type for ordinals below $\omega_{1}$ from the proof of Theorem 4.1. We extend these to ordinals $\omega_{1}<\alpha<\lambda$ as follows: we say $\alpha$ has type $T$ if $\gamma<\omega_{1}$ has type $T$, where $\gamma$ is the unique ordinal such that $|\gamma|_{\prec}=\alpha$. Every $\alpha$ between $\omega_{1}$ and $\lambda$ has type $T$ for some normal type $T$. Also, if $\omega_{1}<\alpha_{1}<\alpha_{2}<\lambda$ both have type $T$, then $\alpha_{1} \in A$ iff $\alpha_{2} \in A$ by indiscernibility. [Say $\alpha_{1}=\left|\gamma_{1}\right|_{\prec}$, where $\gamma_{1}=t(\vec{\beta}, \vec{\varepsilon}), \alpha_{2}=\left|\gamma_{2}\right|_{\prec}$, where $\gamma_{2}=t(\vec{\beta}, \vec{\varrho})$, where $\vec{\beta}<\max (\vec{\alpha})$ and $t$ corresponds to the type $T$. Then $\alpha_{1} \in A$ iff the rank of $t(\vec{\beta}, \vec{\varepsilon})$ in $u\left(\vec{\alpha}, \omega_{1}, \ldots, \omega_{b}\right)$ is in $w\left(\vec{\alpha}, \omega_{1}, \ldots, \omega_{b}\right)$ iff the rank of $t(\vec{\beta}, \vec{\varrho})$ in $u\left(\vec{\alpha}, \omega_{1}, \ldots, \omega_{b}\right)$ is in $w\left(\vec{\alpha}, \omega_{1}, \ldots, \omega_{b}\right)$ iff $\alpha_{2} \in A$.]

It suffices therefore to fix a normal type $T$ (and corresponding term $t$ and ordinals $\vec{\beta}<\max (\vec{\alpha}))$ and show that $X$ is the intersection of a closed and an open set, where $X$ is the set of $\alpha$ between $\omega_{1}$ and $\omega_{2}$ of type $T$. For the rest of the argument we again suppress writing the fixed ordinals $\vec{\alpha}, \vec{\beta}$ and the $\omega_{1}, \ldots, \omega_{b}$. Corresponding to the term $t$ we define the term $t^{\prime}$ by

$$
t^{\prime}\left(\gamma_{1}, \ldots, \gamma_{n}\right)=\left|t\left(\gamma_{1}, \ldots, \gamma_{n}\right)\right|_{\prec}
$$

So, $t^{\prime}$ defines a function from $C^{n}$ to $\lambda$. Since the map $\gamma \mapsto|\gamma| \prec$ is one-to-one, and since $T$ is normal (so $t$ is increasing in each argument), an easy argument shows that $t^{\prime}$ is also increasing in each argument. An analog of Claim 4 holds, namely, there is a permutation $\pi=\left(i_{1}, \ldots, i_{n}\right)$ of $(1, \ldots, n)$ such that for all $\alpha_{1}<\cdots<\alpha_{n} \in C$ and $\beta_{1}<\cdots<\beta_{n} \in C$, we have $t^{\prime}(\vec{\alpha})<t^{\prime}(\vec{\beta})$ iff $\left(\alpha_{i_{1}}, \ldots, \alpha_{i_{n}}\right)<_{\operatorname{lex}}\left(\beta_{i_{1}}, \ldots, \beta_{i_{n}}\right)$ (the difference is that now we do not necessarily have $\left.i_{1}=n\right)$. The proofs of Claims 5 and 6 carry over to $t^{\prime}$ as well. We then define the $A_{j}$ and $F$ exactly as in the proof of Theorem 4.1, and the same proof gives $X=\bar{X}-F$.

As an immediate corollary we have the following. 
Corollary 4.3 (ZF + AD). For any ordinals $\alpha, \beta<\omega_{2}$, $\alpha$ and $\beta$ are Borel isomorphic iff $|\alpha|=|\beta|$.

Theorem 4.2 does not hold for any $\alpha \geq \omega_{2}$ (assuming again AD). This is because there are stationary costationary subsets of $\omega_{2}$, for example, the set of ordinals of cofinality $\omega$.

\section{References}

[1] J. W. Baker, Compact spaces homeomorphic to a ray of ordinals, Fund. Math. 76 (1972), 19-27.

[2] S. Jackson, A new proof of the strong partition relation on $\omega_{1}$, Trans. Amer. Math. Soc. 320 (1990), 737-745.

[3] - Structural consequences of AD, in: Handbook of Set Theory, M. Foreman, A. Kanamori and M. Magidor (eds.), to appear.

[4] T. Jech, Set Theory, 2nd ed., Perspectives in Math. Logic, Springer, 1997.

[5] V. Kieftenbeld and B. Löwe, A classification of ordinal topologies, unpublished notes.

[6] R. D. Mauldin, Countably generated families, Proc. Amer. Math. Soc. 54 (1976), 291-297.

[7] Y. N. Moschovakis, Descriptive Set Theory, Stud. Logic Found. Math. 100, NorthHolland, 1980.

[8] M. B. Rao and K. P. S. B. Rao, Borel $\sigma$-algebra on $[0, \Omega]$, Manuscripta Math. 5 (1971), 195-198.

Department of Mathematics

University of North Texas

P.O. Box 311430

Denton, TX 76203-1430, U.S.A.

E-mail: sgao@unt.edu jackson@unt.edu kieftenbeld@unt.edu

Received 19 January 200\%;

in revised form 8 October 2007 